\title{
Recurrent large pericardial effusion after cardiac surgery presenting with reversible hyponatremia
}

\author{
Sedat Köroğlư ${ }^{1}$, Ahmet Akçay², Hayriye Sayarlıŏglu ${ }^{3}$, Kadir Gişii ${ }^{4}$
}

\footnotetext{
1) Department Of Cardiology, Afsin State Hospital, Kahramanmaras, Turkey

2) Department Of Cardiology, Sutcu Imam University, Kahramanmaras, Turkey

3) Department Of Nephrology, Sutcu Imam University, Kahramanmaras, Turkey

${ }^{4)}$ Department Of Gastroenterology, Sutcu Imam University, Kahramanmaras, Turkey
}

\section{Summary}

\begin{abstract}
Pericardial effusion is a common complication of open heart surgery and more frequently occurs after coronary artery by-pass grafting. There are classical symptoms such as chest pain, dyspnea and orthopnea. However, hyponatremia is an unusual finding on presentation, and reported only three times in the literature. In this paper, a case in which recurrent pericardial effusion developed after coronary artery by-pass grafting presented with reversible hyponatremia was described. Hyponatremia was resolved rapidly, only after removal of large pericardial effusion by a successful pericardiocentesis. The possible mechanisms of reversible hyponatremia, relationship with pericardial effusion, and discrepancies about diagnosis were discussed, briefly.
\end{abstract}

Keywords: Cardiac surgery, hyponatremia; pericardial effusion.

Köroğlu S, Akçay A, Sayarlıŏ̆lu H, Gişi K. Recurrent large pericardial effusion after cardiac surgery presenting with reversible hyponatremia. EJCM 2015; 03 (1): 15-18. DOI: 10.15511/ejcm.14.00118. 


\section{Introduction}

Pericardial effusion is not a rare complication of cardiac surgery and more common in patients undergoing coronary artery by-pass grafting (CABG). ${ }^{(1-3)}$ Patients may present with various symptoms like chest pain, dyspnea, and orthopnea. ${ }^{(4)}$ But presentation with hyponatremia is extremely rare. Here, we described a case with recurrent pericardial effusion after $\mathrm{CABG}$ and its first attack presented with reversible hyponatremia.

\section{Case}

A 55 year old woman was admitted to emergency department with dyspnea on mild exertion, fatigue, abdominal pain, and vertigo. She has undergone CABG operation, two months ago. Past medical history was including hypertension. Her medications were silazapril, acetytsalcilic acid and famotidin.

On physical examination, blood pressure was 100 $160 \mathrm{~mm} \mathrm{Hg}$, heart rate was 84 beats/min, respiratory rate was 18 breaths/min. Heart sounds were distant and deep on cardiac auscultation. There was distension on abdominal examination. Mental status was normal.

Laboratory studies were as follows: Serum sodium, $124 \mathrm{mmol} / \mathrm{L}$; potassium, $4.3 \mathrm{mmol} / \mathrm{L}$; chloride, 90 $\mathrm{mmol} / \mathrm{L}$; blood urea nitrogen, $54 \mathrm{mg} / \mathrm{dl}$; and serum creatinine $1.6 \mathrm{mg} / \mathrm{dl}$. An electrocardiogram showed low voltage on chest leads (Figure 1).

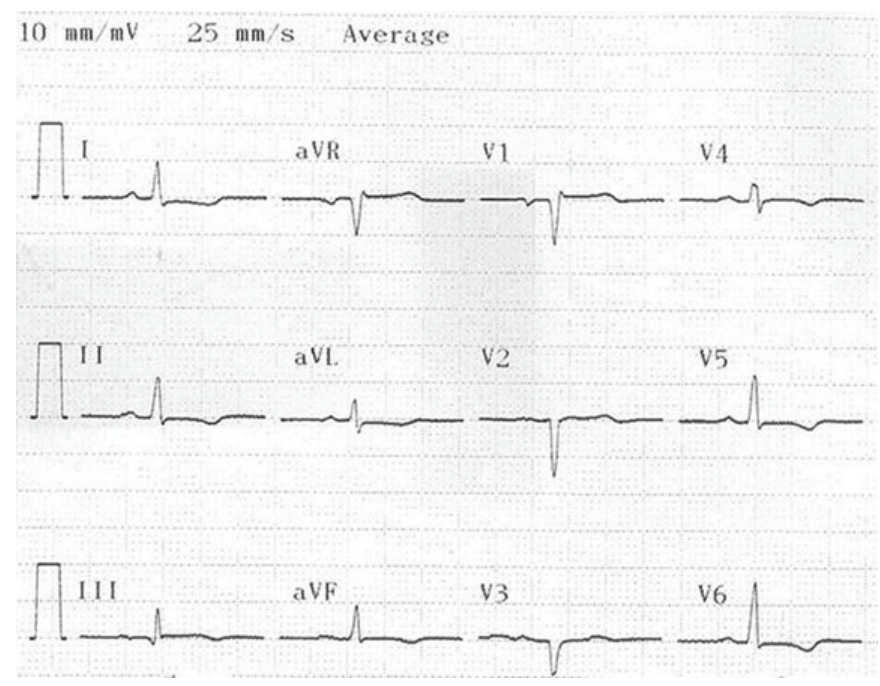

Figure 1. (Legend) The 12-lead electro-cardiogram showing low voltage on chest leads.
Chest radiograph showed cardiomegaly. Transthoracic echocardiogram demonstrated large, diffuse pericardial effusion with evidence of right atrial collapse (Figure 2). Valvular and left ventricular systolic functions were normal.

Because of the progressive symptoms and size of the pericardial effusion pericardiocentesis was performed via subxiphoid route and approximately $1500 \mathrm{ml}$ serous pericardial fluid was removed. A pigtail catheter was leaved in pericardial cavity and during the following 24 hours $1700 \mathrm{ml}$ blood-tinged fluid removed, subsequently. Control laboratory studies were; serum sodium, $134 \mathrm{mmol} / \mathrm{L}$; blood urea nitrogen, $36 \mathrm{mg} / \mathrm{dl}$; serum creatinine, $1.1 \mathrm{mg} / \mathrm{dl}$. Surprisingly, serum sodium was normalized after pericardiocentesis without any specific therapy. In control echocardiography only minimal residual pericardial fluid was detected and patient was discharged with colchicine $0.5 \mathrm{mg} 2 \times 1$ and ibuprofen $800 \mathrm{mg} 2 \mathrm{x} 1$ therapy.

10 days later, patient presented with dyspnea at rest and orthopnea. Echocardiography showed recurrence of large, diffuse pericardial effusion with evidence of right atrial collapse. Serum sodium levels were normal. Pericardiocentesis was performed again, approximately $2500 \mathrm{ml}$ serous pericardial fluid removed and a pigtail catheter was leaved in pericardial cavity. Pericardial

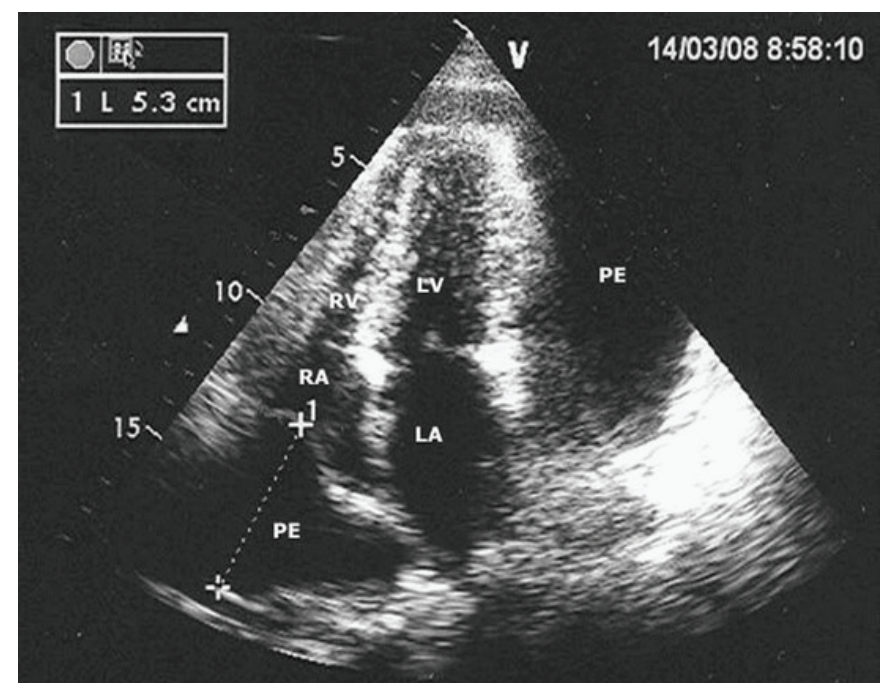

Figure 2. Transthoracic echocardiography from apical 4-chamber view demonstrating large pericardial effusion and right atrial collapse (LA: left atrium, LV: left ventricle, $R A$ : right atrium, $R V$ : right ventricle, $P E$ : pericardial effusion). 
fluid studies were negative for evidence of infectious, neoplastic, or rheumatologic causes.

Pericardial fluid adenosine deaminase level was in normal ranges. During the following 72 hours $2000 \mathrm{ml}$ pericardial fluid removed, subsequently. Two days later, control echocardiography demonstrated recurrence of large, diffuse pericardial effusion again. Owing to the frequent recurrences of large pericardial effusion and risk of tamponade, patient was referred for pericardiopleural fenestration. After a successful operation, she is following up uneventfully for 2 months.

\section{Discussion}

Following cardiac surgery the incidence of pericardial effusion is high (range, 50 to $85 \%$ ) and CABG accounts for more than half of the cases. ${ }^{(1-3)}$ Patients may be asymptomatic or present with various symptoms including chest pain, dyspnea, orthopnea, cough, fatigue and palpitations. ${ }^{(4)}$

Presentation of a pericardial effusion with hyponatremia is extremely rare. In previous reports, inappropriate secretion of antidiuretic hormone as a response to diminished cardiac output in pericardial tamponade was accepted as most plausible mechanism for hyponatremia. ${ }^{(5-7)}$ This response is mediated by baroreceptors in the carotid sinus, which sense a reduction in pressure or stretch, and can overcome the inhibitory effect of hyponatremia on $\mathrm{ADH}$ secretion. ${ }^{(8)}$
Reversibility of hyponatremia and rapid correction after pericardiocentesis without any specific treatment for low serum sodium levels support this hypothesis. After removing the large, diffuse pericardial effusion leading low cardiac output, the cause for secretion of antidiuretic hormone disappeared and serum sodium levels normalized by loosing extra volume.

However, in this case, pericardial effusion was recurrent, resistant to pericardiocentesis and hyponatremia was only detected at the first attack of pericardial fluid accumulation. Serum sodium levels were normal at subsequent episodes of pericardial effusion. Therefore, inappropriate secretion of antidiuretic hormone is not an enough explanation for hyponatremia in this case. Yet, we don't fully know why there was an imbalance between previously mentioned mechanisms in first attack of pericardial effusion and led to hyponatremia, and why there was not for subsequent episodes.

In conclusion, patients with pericardial effusion developed after CABG may present with hyponatremia. Currently, the most reasonable cause of hyponatremia seems to the imbalance between the antinatriuretic and antidiuretic responses to pericardial effusion leading to low cardiac output. Hyponatremia resolves, only when the effusion removed without any specific therapy. Further investigations are needed to understand all factors contributing hyponatremia in patients with pericardial effusion. 


\section{References}

1. Weitzman LB, Tinker WP, Kronzon I, Cohen ML, Glassman E, Spencer FC. The incidence and natural history of pericardial effusion after cardiac surgery--an echocardiographic study. Circulation 1984;69:506-11.

2. Ikäheimo MJ, Huikuri HV, Airaksinen KE, Korhonen UR, Linnaluoto MK, Tarkka MR, et al. Pericardial effusion after cardiac surgery: incidence, relation to the type of surgery, antithrombotic therapy, and early coronary bypass graft patency. Am Heart J 1988;116:97-102.

3. Pepi M, Muratori M, Barbier P, Doria E, Arena V, Berti M, et al. Pericardial effusion after cardiac surgery: incidence, site, and hemodynamic consequences. Br Heart $J$ 1994;72:327-31.

4. Shahbaz Sarwar CM, Fatimi S. Characteristics of recurrent pericardial effusions. Singapore Med J 2007;48:725-8.

5. Groves PH, Shah AM, Hutchison SJ. Hyponatremia secondary to an inappropriately high release of antidiuretic hormone in cardiac tamponade. Br Heart J 1990;64:206-7.

6. Mouallem M, Wolf I, Mindlin G, Farfel Z. Pericardial tamponade-associated hyponatremia. Am J Med Sci 2003;325:51-2.

7. Shafique R, Sarwar S, Wall BM, Cooke CR. Reversible hyponatremia related to pericardial tamponade. Am J Kidney Dis 2007;50:336-41.

8. Raff H, Cogswell TL, Bernath GA, Klopfenstein HS. Vasopressin and ACTH responses to acute cardiac tamponade in conscious dogs. Clin Res 1986;34:899.

Received: 07/11/2014

Accepted: 18/02/2015

Published: 15/06/2015

Disclosure and conflicts of interest:

Conflicts of interest were not reported.

\section{Corresponding author:}

Dr. Sedat Köroğlu

m.sedatkoroglu@gmail.com 